\title{
Estimation of the transmission dynamics of African swine fever virus within a swine house
}

\author{
J. P. NIELSEN ${ }^{1} \dagger$, T. S. LARSEN ${ }^{1} \dagger$, T. HALASA ${ }^{2}$ AND L. E. CHRISTIANSEN ${ }^{1 *}$ \\ ${ }^{1}$ Dynamical Systems, Department of Applied Mathematics and Computer Sciences, Technical University of \\ Denmark, Lyngby, Denmark \\ ${ }^{2}$ Division of Diagnostics and Scientific Advice, National Veterinary Institute, Technical University of Denmark, \\ Lyngby, Denmark
}

Received 31 March 2017; Final revision 21 June 2017; Accepted 9 July 2017; first published online 3 August 2017

\section{SUMMARY}

The spread of African swine fever virus (ASFV) threatens to reach further parts of Europe. In countries with a large swine production, an outbreak of ASF may result in devastating economic consequences for the swine industry. Simulation models can assist decision makers setting up contingency plans. This creates a need for estimation of parameters. This study presents a new analysis of a previously published study. A full likelihood framework is presented including the impact of model assumptions on the estimated transmission parameters. As animals were only tested every other day, an interpretation was introduced to cover the weighted infectiousness on unobserved days for the individual animals (WIU). Based on our model and the set of assumptions, the within- and between-pen transmission parameters were estimated to $\beta_{\mathrm{w}}=1.05(95 \%$ CI $0.62-1 \cdot 72), \beta_{\mathrm{b}}=0.46(95 \%$ CI $0 \cdot 17-1 \cdot 00)$, respectively, and the $\mathrm{WIU}=1.00(95 \%$ CI $0-1)$. Furthermore, we simulated the spread of ASFV within a pig house using a modified SEIR-model to establish the time from infection of one animal until ASFV is detected in the herd. Based on a chosen detection limit of $2.55 \%$ equivalent to 10 dead pigs out of 360 , the disease would be detected 13-19 days after introduction.

Key words: African swine fever, epidemiology, maximum likelihood, modelling, transmission.

\section{INTRODUCTION}

African swine fever (ASF) is a notifiable infectious disease in pigs (cited from [1]). It is caused by ASF virus (ASFV), a DNA virus from the family Asfarviridae, genus Asfivirus [2]. Since the introduction of ASFV to Georgia in 2007, it has spread to the Russian Federation and several Eastern EU countries, including Latvia, Estonia, Lithuania, Moldova and Poland [3,4].

\footnotetext{
* Author for correspondence: L. E. Christiansen, DTU Compute, Building 324, DK-2800 Kgs., Lyngby, Denmark.

(Email: laec@dtu.dk)

$\dagger$ They are joint first authors..
}

ASF is considered to be a substantial threat for Western Europe [5]. In countries with a large production and/or export of swine and swine products, an outbreak of ASF may result in devastating economic consequences for the swine industry due to export restrictions, as exemplified by the Danish situation $[1,6]$.

Simulation models are widely used to study the spread of animal diseases within a country and to propose effective control strategies to limit their spread [1,7-13]. An important element of these spread models is modelling the progress of the disease within the unit of interest, i.e. the pig house. Understanding this mechanism is important as it may have substantial impact on virus 
spread to other herds and disease detection within the infected herds.

The ASFV spreads mainly due to direct contact between infectious and susceptible animals [14-16]. It can also spread via indirect and airborne contact $[15,17]$. Quantifying the virus spread potential via each route is important for proper modelling of the spread of the virus within the herd. Using data from a pig-to-pig transmission study [14], Guinat et al. [15] estimated the transmission parameters of ASFV via the direct and indirect routes, separately, within a pig house. The underlying assumptions behind the estimated transmission parameters could have impacted the estimated values. This warrants an investigation of the impact of the chosen assumptions on the estimated values of the transmission parameters.

In the current study, we estimated the transmission parameters for the different routes of virus spread based on the same data [14] and demonstrated the impact of model assumptions on the estimated transmission parameters. In addition, we simulated the spread of ASFV within a pig house using the estimated parameters to establish the time from the infection of one animal until ASFV is detected in the herd.

\section{METHODS}

\section{Data}

The data originates from Guinat et al. [14] where experiments were carried out on 40 weaner pigs at the age of 7 weeks positioned in four separate rooms $A, B, C$ and $D$. In each of these rooms, a specific number of randomly chosen pigs were inoculated intramuscularly with the virulent Georgia 2007/1 ASFV strain.

In rooms $A$ and $D$, containing, respectively, 10 and six pigs, half of the pigs were inoculated on day 0 . Rooms $B$ and $C$ were each divided into two adjacent pens, pen 1 and pen 2 , separated by a fence under which it was possible for small amounts of urine and faeces to pass. Direct nose-to-nose contact was not possible. Eight pigs were located in pen 1, and on day 0 half of these were initially inoculated. In the adjacent pen 2, four pigs were situated. This provided a situation with direct contact for the pigs in rooms $A$ and $D$, and both direct and indirect contact for the pigs in rooms $B$ and $C$.

Infection was considered to have occurred when a blood sample, tested by virus isolation and quantitative real-time polymerase chain reaction (qPCR), was confirmed positive for the virus; as considered in the original study [14]. Samples were collected every other day starting from day 3 . For further details on the sampling, we refer to Guinat et al. [15].

\section{Model}

To model the spread of ASFV, a modified version of the SEIR model was chosen [18]. In this model, the animals are assigned to four compartments depending on their health state. The susceptible animals, which initially counted all non-inoculated pigs in the experiment, were located in the first compartment $(S)$. When an animal became infected, it moved to the exposed compartment $(E)$. Animals remained infected for a latent period of $L$ days after which they became infectious and moved to compartment $(I)$. After an infectious period of $T$ days, the pigs entered the recovered compartment $(R)$ - either dead or immune. However, all pigs were euthanized for welfare reasons [14].

It was assumed that the number of pigs, becoming infected at time $t$ throughout the time period $\Delta t$ in pen $i\left(E_{i, t}\right)$, out of the total amount of susceptible pigs $\left(S_{i, t}\right)$, was binomially distributed with a certain probability of a single pig becoming infected, $p_{i, t}$. This probability of becoming infected depended on the total number of animals $\left(N_{i, t}=S_{i, t}+E_{i, t}+I_{i, t}\right)$, the number of infectious pigs $\left(I_{i, t}\right)$, and their number of infectious contacts with the remaining susceptible pigs within and between pens $\left(\beta_{\mathrm{w}}\right.$ and $\beta_{\mathrm{b}}$ ) [19]. The probability of avoiding becoming infected was obtained from the probability density function of the Poisson distribution [20]. To find the actual probability of becoming infected, this was subtracted from 1 . Therefore, the probability of infection in each pen was given by:

$$
\begin{aligned}
& p_{1, t}=1-\exp \left(-\left(\beta_{\mathrm{w}} \frac{I_{1, t}}{N_{1, t}}+\beta_{\mathrm{b}} \frac{I_{2, t}}{N_{1, t}+N_{2, t}}\right) \Delta t\right) \text { and } \\
& p_{2, t}=1-\exp \left(-\left(\beta_{\mathrm{w}} \frac{I_{2, t}}{N_{2, t}}+\beta_{\mathrm{b}} \frac{I_{1, t}}{N_{1, t}+N_{2, t}}\right) \Delta t\right) .
\end{aligned}
$$

From the assumption that the data are binomially distributed, the parameters $\beta_{\mathrm{w}}$ and $\beta_{\mathrm{b}}$ were estimated using the maximum-likelihood method. In Guinat et al. [15], the 95\% confidence intervals (95\% CIs) for the transmission parameters were determined by adding \pm 1.96 times the standard error to the estimates. This approach provides symmetric CIs, corresponding to an underlying assumption of normality of the estimated parameters, this assumption is often violated when parameters are bounded as in the 
present case where both $\beta_{\mathrm{w}}$ and $\beta_{\mathrm{b}}$ have to be positive. Therefore, to estimate the corresponding 95\% CIs, we chose to use the profile likelihood method, which has the advantage of allowing asymmetric CIs [21].

The parameter estimation was initially done for different values of $L$. We have chosen to consider latent periods of 3, 4 and 5 days. These three values of $L$ were chosen as pigs infected by intramuscular inoculations showed clinical signs $4 \cdot 4 \pm 1 \cdot 0$ days after inoculation [14].

We were also interested in determining if one of the three durations of $L$ was more likely or if there was a general variation within the tested animals. Therefore, a new model using the rule of average conditional probabilities was constructed [22]. This allowed us to adjust the current model to a new joint model that considered $L$ as a stochastic variable. The rule of conditional probabilities is given by the formula:

$P(Y=y)=\sum_{z} P(Y=y \mid Z=z) P(Z=z)$.

The conditional probability $P(Y=y \mid Z=z)$ is the probability of an event $y$ given $Z=z$. We expressed this in terms of $L$ and the data observations $x_{i}$ and inserted the expression into the likelihood function. The likelihood was expressed as a function of $\beta_{\mathrm{w}}, \beta_{\mathrm{b}}$ and the probability of each $L$ :

$\mathcal{L}\left(x_{i} ; \beta_{\mathrm{w}}, \quad \beta_{\mathrm{b}}\right)=\prod_{i=1}^{M} \sum_{l=3}^{5} P\left(X=x_{i} \mid L=l\right) P(L=l)$.

Here $x_{i}$ are the data observations and $M$ is the number of days with observations. As before, the infection parameter estimates were determined using maximum likelihood. However, in this model, the probabilities of the specific duration of latent period could be determined as well.

When possible, models are compared by using a likelihood ratio test [21], which determines if parameters from one model are significantly different from parameters in another nested model. If this is not possible, the models can be compared based on their Akaike's Information Criterion (AIC).

MATLAB and Statistics Toolbox Release 2015b, The MathWorks, Inc., Natick, Massachusetts, USA, was used in this study.

\section{Assumptions}

When estimating the transmission parameters, it was necessary to establish when each pig became infected and when it got infectious. When doing this, only the naturally infected pigs were considered. We also had to determine the number of infectious pigs that could have transmitted the disease. It was assumed that euthanized pigs were infectious too, including the day of euthanasia and that no pigs were infectious on day 2 .

The particular day a pig was infected was calculated by subtracting the number of days in $L$ from the day in the experiment when the pig became infectious. However, as the observations were only noted on every other day, this provided a time interval of 2 days for becoming infectious, and therefore also a 2-day interval for becoming infected. For this particular reason, an interpretation was used to cover the weighted infectiousness on unobserved days for the individual animals (WIU). It is not specified how this matter was handled in Guinat et al. [15] and hence we were unable to reproduce the results from the article. Therefore, we chose to introduce the parameter WIU.

WIU was treated as a continuous variable in the range $[0,1]$. On days where an animal was not observed, WIU $=0$ indicates that all pigs have the same infectiousness status as the day before. Similarly, WIU $=1$ indicates that all pigs have the same infectiousness status as the following day. Since no animals recover during the experiment, then WIU only affects when an animal converts from being infected to also becoming infectious.

To be able to compare the parameter estimates, it was necessary for them to be based on the same observations for each $L$, as the maximum-likelihood method depends on this. Since our intention was to compare the maximum log likelihood values directly, we excluded the observations on day 7 for all $L$, as choosing the latent period $L=5$ would require observations on day 2 that were not available.

\section{Simulations}

The obtained estimates of the transmission parameters allowed us to make stochastic simulations of the spread of ASFV within a pig house. We did this in order to examine if a time frame for detection of an outbreak could be established.

We based our simulations on the SEIR model, where the pigs were located in one of the compartments $S, E, I$ or $R$. Initially all pigs, except the one pig initiating the outbreak, were located in compartment $S$. During the time for the first infectious pig to 
reach compartment $R$, the infection would either die out or spread to the rest of the herd.

We chose to consider a housing structure containing six adjacent pens on each side of a passage. Each pen contained 30 pigs. Due to symmetry, this left us with three different points of initial spread represented by initial spread in pens 1,2 and 3 respectively. A thousand independent outbreaks were simulated and for each of these the transmission parameters $\beta_{\mathrm{w}}$ and $\beta_{\mathrm{b}}$ were sampled from two independent zero-truncated normal distributions. All parameters, including the latent period, were based on the model that maximizes the likelihood of the data. To ensure that the pigs stayed in the exposed state for a given number of days, compartment $E$ was divided into a corresponding number of subcompartments with a probability of $100 \%$ of moving to the next compartment the following day.

We chose to consider the infectious period from Guinat et al. [14] with a mean of $4 \cdot 5$ days and a standard deviation of 0.75 days. To make sure that the infectious pigs remained infectious for at least 3 days, corresponding to the lower limit of the $95 \%$ CI, compartment $I$ was divided into three subcompartments. The probability of moving from one sub-compartment to the next was set to $2 / 3$, and the rates $r_{I_{1} I_{2}}, r_{I_{2} I_{3}}$ and $r_{I_{3} R}$, where $r_{A B}$ denotes the number of pigs moving from compartment $A$ to $B$, were found as independent random samples from a binomial $(m$, $2 / 3$ ) distribution. Here $m$ is the number of infectious pigs in sub-compartment $A$. This means that the infectious period of each animal will follow an Erlang distribution with $k=3$ and $\lambda=(2 / 3)$. The mean of this duration is 4.5 days and the standard deviation for the individual animals is 2.6 days. It is unknown if Guinat et al. [14] used a fixed duration for all animals. The initial infectious pig was placed in the first subcompartment of $I$.

Since the chosen housing structure differed from the one used in the experimental setup, the formulation of the probability of infection is not directly transferrable. From the assumption that spread of infection is faster to the closest neighbours, we now considered an individual probability formulation for each pen depending on its location and the distance to its neighbours. It was assumed that nose-to-nose contact could only occur between pigs within the pen. The distance between two neighbour pens was set to $d=1$, and the passage was accounted for by adding the constant $\delta=$ 1. Finally, $\rho$ was a weight between 0 and 1 , describing how fast the force of infection decreased. As an example, we considered the probability of infection given in pen 1:

$$
\begin{aligned}
p_{1, t}= & 1-\exp \left(-\left(\beta_{\mathrm{w}} \frac{I_{1, t}}{N_{1, t}}\right.\right. \\
& \left.\left.+\beta_{\mathrm{b}} \frac{\sum_{i=1}^{5} \rho^{i} I_{1+i}+\sum_{i=1}^{6} \rho^{i+\delta} I_{6+i}}{\sum_{i=1}^{6} \rho^{i} N_{i}+\sum_{i=1}^{6} \rho^{i+\delta} N_{6+i}}\right)\right),
\end{aligned}
$$

$\rho^{i}$ were the weights of the pens on the same side of the passage as pen 1 , and $\rho^{i+\delta}$ were the weights of the pens on the other side of the passage. The remaining 11 probabilities were formulated in a similar fashion. Therefore, the number of pigs in pen 1, moving from compartment $S$ to the first sub-compartment of $E$ and given as the rate $r_{S E_{1}}$, was found as a random samples from a $\left(s, p_{1, t}\right)$-binomial distribution. Here $s$ is the number of pigs in compartment $S$.

We estimated the time for detection of the disease, within the pig house, based on the number of dead animals. Halasa et al. [1] estimated this mortality level due to ASF when the disease was detected within a large infected herd. Detection occurred when the mortality was $2.55 \%$. In our simulation, this is equivalent to at least 10 out of 360 animals in the house. We also examined the time for detection using 5 or 15 dead animals, respectively.

\section{RESULTS}

\section{Parameter estimation}

When fixing the latent period, it can be seen from Table 1 that a latent period of 5 days would be preferred as it provides the highest maximum likelihood of -32.64 and hereby also the lowest AIC with the parameter estimates $\beta_{\mathrm{w}}=1.05 \quad(0.62-1.72), \quad \beta_{\mathrm{b}}=0.46$ $(0 \cdot 17-1 \cdot 00)$ and WIU $=1 \cdot 00(0-1)-$ the numbers in parentheses are the $95 \%$ CIs. When comparing the other durations of the latent period, the transmission parameters decrease as the duration of the latent period decreases. However, the only case where the estimate of WIU is different from 1.00 is for $L=4$. It should be noted that the $95 \%$ CIs for the WIU are $(0-1)$ in every case (Table 1). Every value of $\beta_{\mathrm{w}}$ is included in the CIs for the remaining $\beta_{\mathrm{w}}$ values. Furthermore, for each $L$ the CIs for $\beta_{\mathrm{w}}$ and $\beta_{\mathrm{b}}$ intersect.

When allowing a mixture of the latent periods, the maximum-likelihood estimates can be found in Table 2. Here, a latent period of 5 days was the most likely period with a probability of $P_{L=5}=71.62 \%$. Again, WIU was estimated to be $1 \cdot 00(0-1)$ and the transmission parameter estimates were $\beta_{\mathrm{w}}=1.04$ 
Table 1. Parameter estimates (95\% confidence intervals) for the within-pen $\left(\beta_{w}\right)$ and between-pen $\left(\beta_{b}\right)$ transmission rate, weighted infectiousness of unobserved days for the individual animals (WIU), maximum-likelihood values $\left(\max \mathcal{L}_{\log }\right.$ ) and the AIC estimated using a latent period $(L)$ of 3,4 or 5 days

\begin{tabular}{llll}
\hline \hline Parameter & $L=3$ & $L=4$ & $L=5$ \\
\hline$\beta_{\mathrm{w}}$ & $0 \cdot 95(0 \cdot 57-1 \cdot 47)$ & $1 \cdot 00(0 \cdot 56-1 \cdot 69)$ & $1 \cdot 05(0 \cdot 62-1 \cdot 72)$ \\
$\beta_{\mathrm{b}}$ & $0 \cdot 31(0 \cdot 08-0 \cdot 74)$ & $0 \cdot 46(0 \cdot 16-1 \cdot 06)$ & $0 \cdot 46(0 \cdot 17-1 \cdot 00)$ \\
WIU & $1 \cdot 00(0-1)$ & $0.56(0-1)$ & $1 \cdot 00(0-1)$ \\
$\max \mathcal{L}_{\log }$ & $-33 \cdot 51$ & $-33 \cdot 61$ & $-32 \cdot 64$ \\
AIC & $73 \cdot 01$ & $73 \cdot 22$ & $71 \cdot 28$ \\
\hline \hline
\end{tabular}

Table 2. Average conditional probabilities

\begin{tabular}{llllllll}
\hline \hline$\beta_{\mathrm{w}}$ & $\beta_{\mathrm{b}}$ & $P_{L=3}$ & $P_{L=4}$ & $P_{\mathrm{L}=5 ;}$ & WIU & $\max \mathcal{L}_{\log }$ & AIC \\
\hline $1 \cdot 04(0 \cdot 61-1 \cdot 65)$ & $0 \cdot 40(0 \cdot 10-0 \cdot 95)$ & $28 \cdot 38 \%(0-100)$ & $0 \cdot 00 \%(0-100)$ & $71 \cdot 62 \%(0-100)$ & $1 \cdot 00(0-1)$ & $-32 \cdot 53$ & $75 \cdot 06$ \\
\hline \hline
\end{tabular}

\begin{tabular}{|l|l|l|l|l|l|}
\hline 1 & 2 & 3 & 4 & 5 & 6 \\
\hline 7 & 8 & 9 & 10 & 11 & 12 \\
\hline
\end{tabular}

Fig. 1. Contour plot showing the asymmetric $95 \%$ confidence intervals in bold corresponding to a latent period of 5 days (Table 1).

$(0 \cdot 61-1 \cdot 65)$ and $\beta_{\mathrm{b}}=0 \cdot 40(0 \cdot 10-0 \cdot 95)$, resembling the values in Table 1 for $L=5$. It should be noted that the probability of having a latent period of 4 days is estimated to 0. As expected, the maximum-likelihood value was highest for this model, compared with the maximum-likelihood values in Table 1, when the latent period was fixed to 5 days.

A likelihood ratio test was performed in order to test if a mixture of the latent period (Table 2) is significantly better than a fixed duration of the latent period (Table 1). As the biggest difference in maximum-likelihood values is between the one from Table 2 and the one for $L=4$ from Table 1 , we started by testing only these two models using the likelihood ratio test. This yielded a $P$-value of $0 \cdot 3396$. Since this $P$-value is $>0.05$, we cannot, with a significance level of 0.05 , reject the null hypothesis, i.e. we do not have evidence to conclude that the estimates in Table 2 are more likely than the estimates in Table 1. Thus, based on the current data, a fixed latent period of 5 days is the most likely latent period, and it is significantly better than using a mixture of integers for the latent period.

As mentioned earlier, the profile likelihood method has the advantage of allowing asymmetric CIs.
Figure 1 shows that the CIs for the estimated transmission parameters for $L=5$ in Table 1 are asymmetric as they do not form an ellipse. This confirms the necessity of using a method that allows asymmetric CIs such as the profile likelihood method.

\section{Simulations}

When carrying out the simulations, a value of $\rho$, the weight of the pen for infection spread, must be chosen. However, we do not have any experimental evidence pointing towards a specific value - except that it should be positive and less than one. We have chosen to examine $\rho=0.5, \rho=0.7$ and $\rho=0.9$ to span a wide range.

Figure 2 shows the accumulated number of dead pigs on each day including percentiles. It can be seen that the curves are steeper the higher the value of $\rho$, indicating a faster spread of the virus. Therefore, we can see that the value of $\rho$ influences the simulation results. Detailed within house outbreak data could be used to estimate $\rho$ or suggest alternative formulations. We have chosen to use $\rho=0 \cdot 7$ in the remaining simulations.

Figure 3 shows the median number of accumulated dead pigs for each pen. It is seen that the distance between the pens influences the timing of the infection. First of all, infection takes off about 10 days earlier in the pen with the initially infectious pig compared with infection in the remaining pens. When inspecting the ordering of the other pens, it becomes clear that those closer to the initially infectious pen get infected before those further away. 


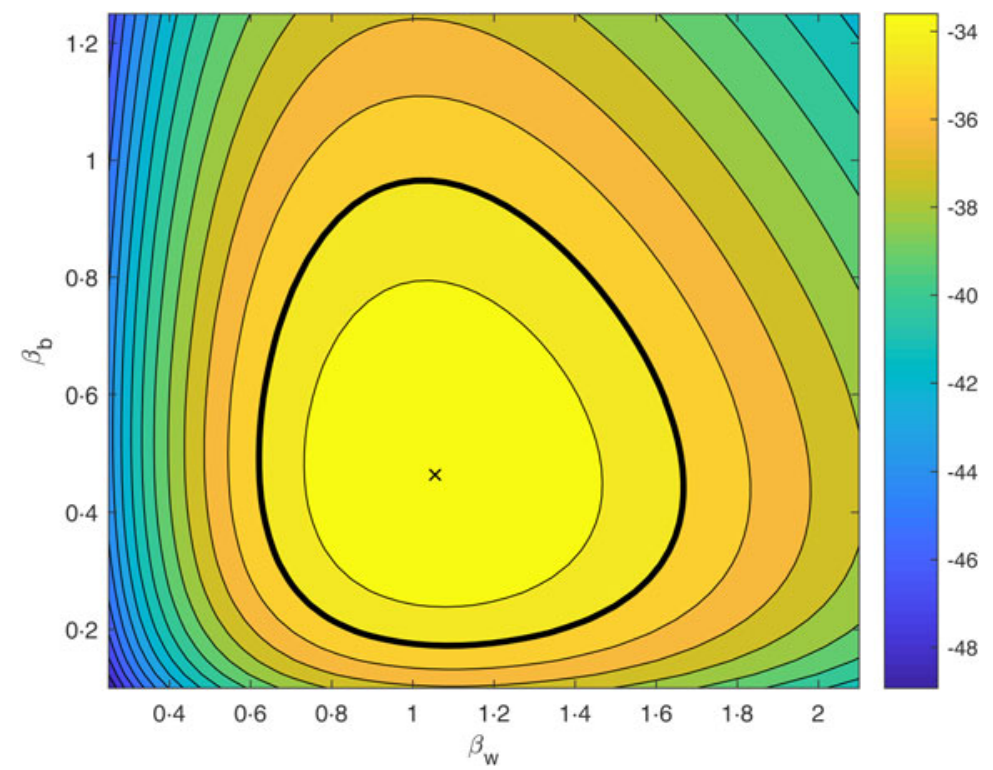

Fig. 2. Accumulated number of dead pigs with the initially infected pig located in pen $1 .(a) \rho=0 \cdot 5 \cdot(b) \rho=0 \cdot 7$. $(c) \rho=0 \cdot 9$. The simulations are represented by the median and the $50 \%(25 \% ; 75 \%)$ and $95 \%(2 \cdot 5 \% ; 97 \cdot 5 \%)$ simulation envelopes.

Figure 4 shows that in the majority of the simulations, the entire herd will eventually become infected and die. However, it is also seen that in some cases the disease will die out before the infection spreads further.

When looking at the median for the 1000 simulations, we see that five pigs had died on day 13 after introduction, independently on the location of the initially infectious pig, day 17 was the first day where at least 10 pigs had died in both cases, and 15 pigs were dead, respectively, 19 and 18 days after introduction of infection. Therefore, based on the chosen detection limit, the disease will be detected 13-19 days after introduction. This confirms the results on Figure 4 showing no apparent difference in the timing of the infection based on the initial location of the infection.

\section{DISCUSSION}

\section{Parameter estimation}

In this study, within- and between-pen transmission parameters for the spread of ASFV in a pig house were estimated. Based on maximum-likelihood estimation and likelihood ratio testing, a fixed latent period of 5 days was found to most adequately describe the data (Table 1). Under more realistic conditions, the force of infection between pigs in ordinary pig houses may vary more, and thus we expect that the latent period should be described by a distribution.
However, the current data did not contain enough information to confirm this. Thus, more studies are needed, i.e. preferably with detailed observations from an outbreak in a real pig house.

Some of the data observations used in this study made it unlikely to have a WIU close to zero, i.e. the same status as the day before. This is especially clear on day 10 in room $A$ and on day 8 in room $D$ when considering $L=3$, e.g. in room $D$ there are no infectious animals on day 8 if WIU $=0$, but on day 11 , corresponding to $L=3$, two newly infected animals are diagnosed. In these cases, setting WIU close to zero would mean that several pigs got infected even though the risk of becoming infected was very small. This pushes WIU away from zero and towards one. In general, it is clear that a higher WIU will increase the probability for a susceptible pig to become infected as pigs are considered to become infectious sooner. This may be counterbalanced by a longer latent period, and thus it is not surprising that $L=5$ leads to WIU $=1$.

The estimate of WIU is 1 for $L=3$ and $L=5$, but for $L=4$ WIU is $<1$. This could be due to the fact that animals are tested every other day. If ethically possible, it would be better to use a dynamic sampling strategy with more frequent sampling in periods with higher risks.

In the mixture model for the latent period, the estimated probability of $L=4$ was zero. As seen above, 

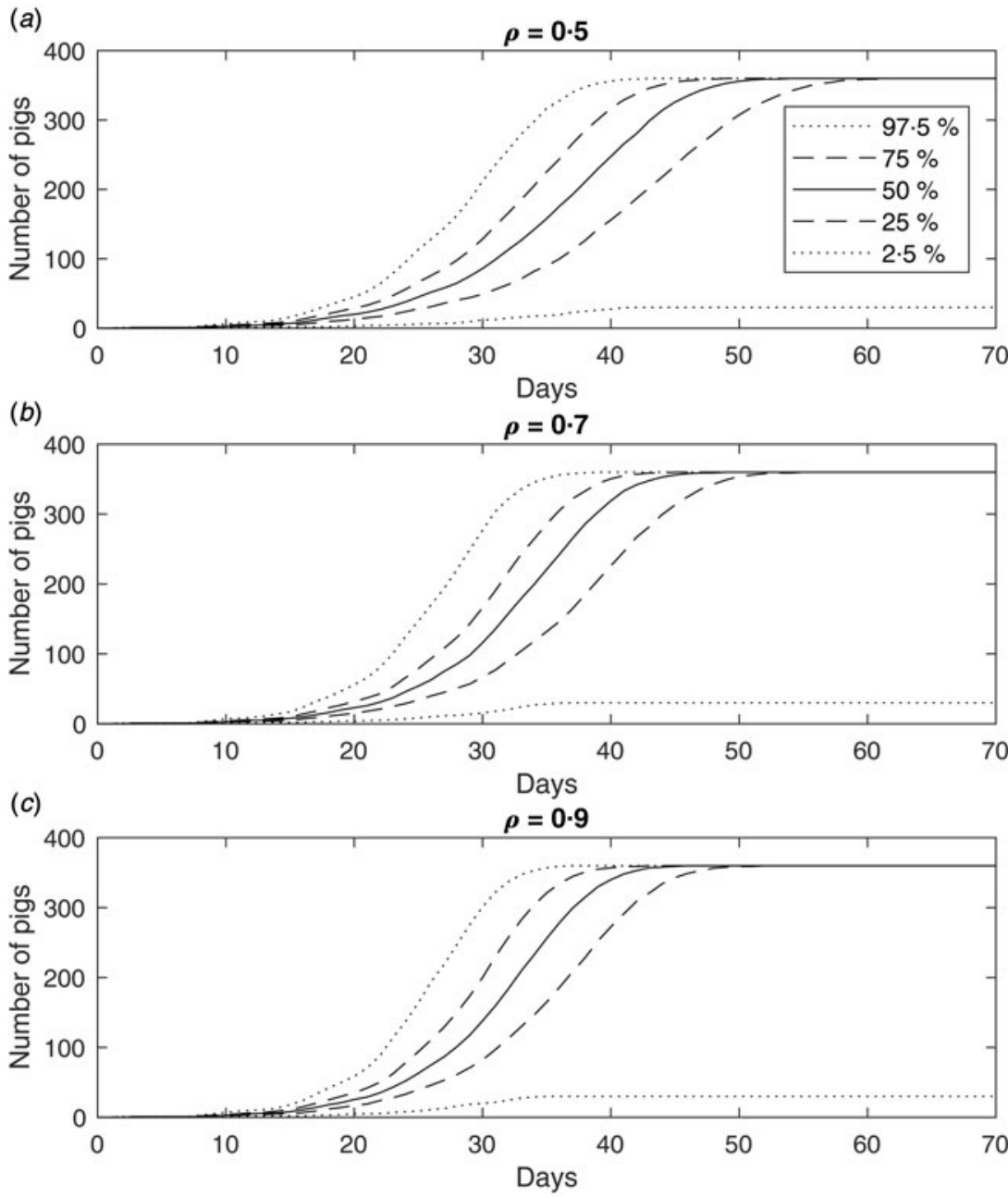

Fig. 3. The median of the accumulated number of infectious pigs in each pen depending on the location of the initially infectious pig. (a) The infectious pig is located in pen 1. (b) The infectious pig is located in pen 3.

there are particular animals where $L=3$ or $L=5$ is more likely. It is believed that those particular observations have a high influence on the likelihood function, and thus push the probability mass away from $L=4$.

In the study of Guinat et al. [15], there are no comments regarding the issue of day 7; when a latent period of 5 days is assumed, observations on day 7 cannot be used on the same terms as when a latent period of 3 or 4 days is used. The estimated parameters can be based on 18 observations for a latent period of 3 or 4 days and only 14 observations for a latent period of 5 days. The maximum-likelihood method is very sensitive to this problem as the maximum-likelihood value in general becomes lower when it is based on more observations. This means that to be able to compare all of the maximum-likelihood values, the calculation has to be done based on the same observations.
This problem is not being addressed in [15], and therefore the reported models 1 and 2 cannot be directly compared with model 3 , as is the case. It turns out that excluding the four observations, making it possible to compare all three models, changes the parameter estimates considerably (data not shown). This can be explained by the fact that these four observations occur in the first couple of days in the experiment, where few animals are infected, leading to a lower risk of infection. Furthermore, it could be due to an increase in infectiousness for the infectious animals after the initial detection by qPCR.

As mentioned earlier, the $95 \%$ CIs for $\beta_{\mathrm{w}}$ and $\beta_{\mathrm{b}}$ overlap for each $L$. This made us consider an alternative one parameter model (data not shown). Our primary goal with this model was to examine whether a model including only airborne transmission was more likely than a model including both airborne and direct transmission. 


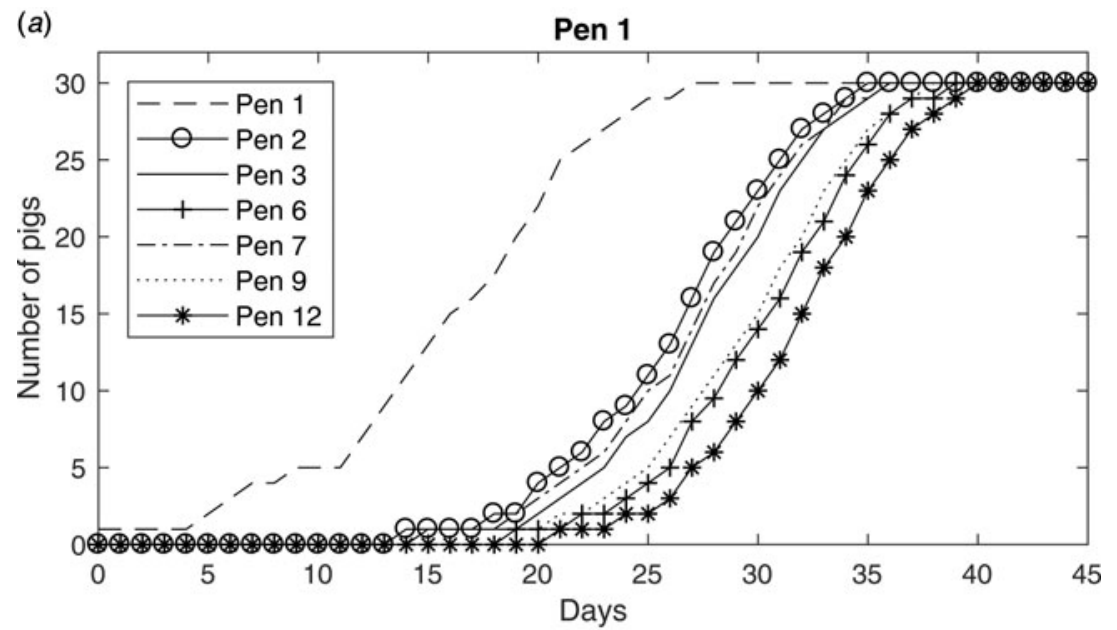

(b) Pen 3

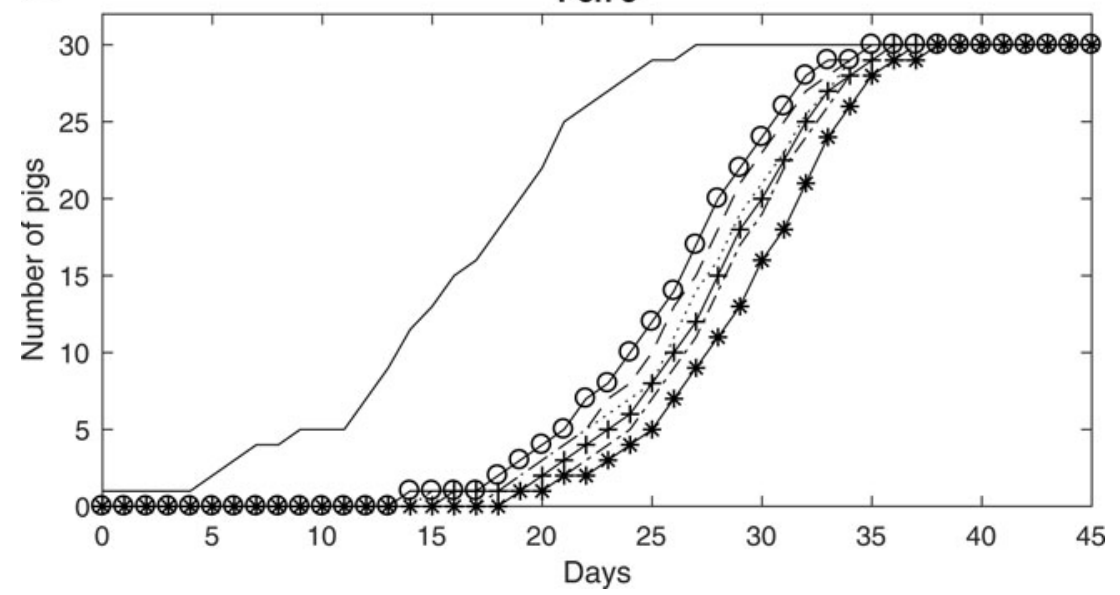

Fig. 4. Accumulated number of infectious pigs and accumulated number of dead pigs depending on the location of the initially infectious pig. (a) The infectious pig is located in pen 1. (b) The infectious pig is located in pen 3 . The simulations are represented by the median and the $50 \%(25 \% ; 75 \%)$ and $95 \%(2 \cdot 5 \% ; 97 \cdot 5 \%)$ simulation envelopes.

The likelihood ratio test indicated that a model including both airborne and direct transmission is more likely than a model including only airborne transmission (results not shown). This alternative formulation is not nested within the model presented in this paper, so they cannot be compared with a likelihood ratio test. However, they can be compared using AIC, and as the two model structures have the same number of parameters, then this corresponds to preference for the model with the highest likelihood. The likelihood is higher for the model presented in this paper (Table 1), and thus the presented model remains preferred.

Guinat et al. have made a corrigendum [23], and for a latent period of 5 days, it is to some degree possible to compare their findings with this paper. The estimated transmission parameters are similar. However, the CIs are markedly different as expected due to the difference in methodology. De Carvalho Ferreira et al. [24] estimated the within-pen transmission rate for the Malta '78 and the Netherlands ' 86 ASFV isolates. The estimates varied from 0.45 and 3.63 (mean values) depending on the infection dose of the inoculated animals and the criteria of infectiousness and moment of infection that was used to calculate the transmission rate. In the current study, the estimates of the within-pen transmission rate for the Georgia 2007 strain are close to 1 and thus within the range found by de Carvalho Ferreira et al. Actually, the $95 \%$ CIs are also within the previously described range.

\section{Simulations}

Guinat et al. [15] simulated a circular pig house with independent contacts to both neighbours. We found 
(a)

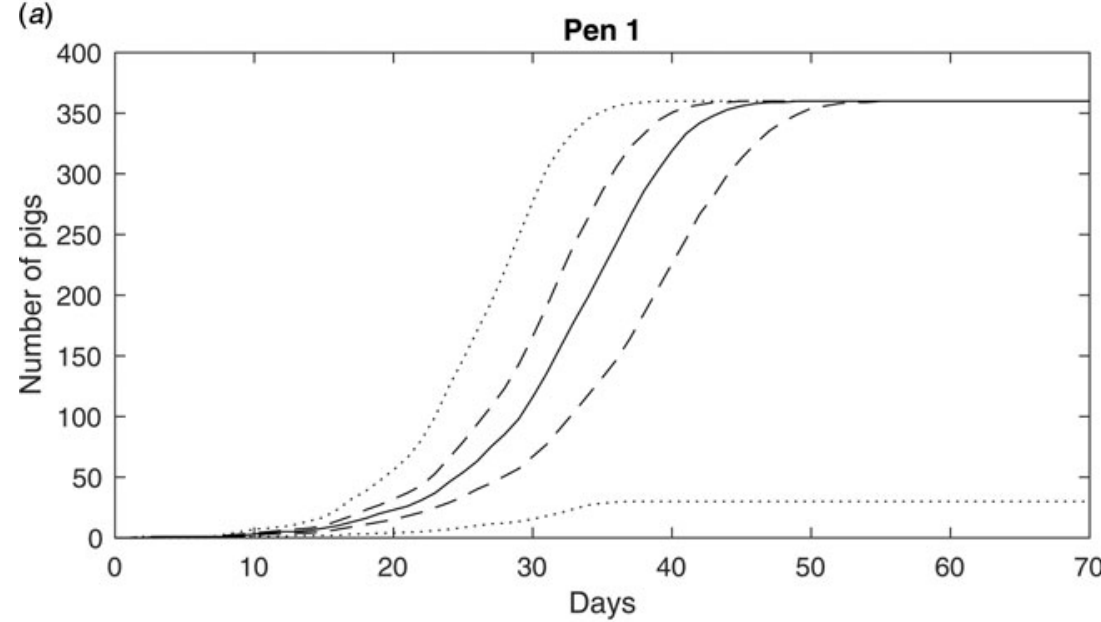

(b)

Pen 3

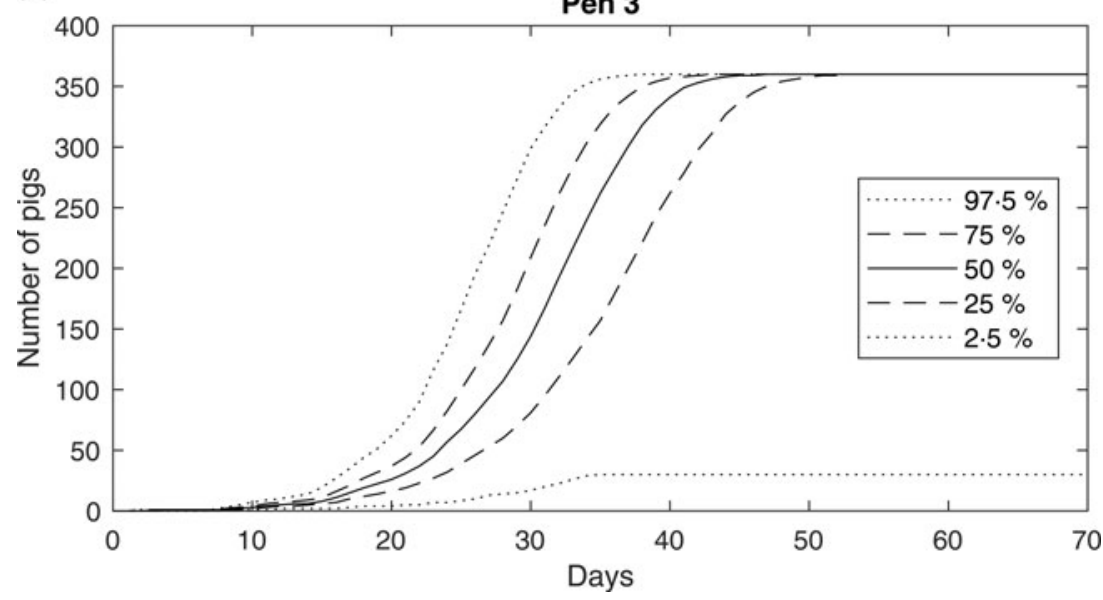

Fig. 5. The proposed housing structure showing a total of 12 pens in a pig house.

this structure to be artificial and with an increased force of infection. Hence, we simulated another structure (Fig. 5) with a more realistic resemblance of a typical structure of a Danish pig house. For a moderately contagious disease like ASF [25], the structure of the housing system may play a role in the spread of the virus, and hence the detection of the disease within the herd.

We studied a possible detection limit for the farmer, based on the total number of infectious pigs showing clinical symptoms and the total number of dead pigs. As our limits are merely suggestions and the attentiveness of the farmers varies, other detection parameters could be examined as well. For instance, the farmer could also suspect an outbreak of the virus if, on a single day, the number of dead pigs or pigs showing clinical symptoms increased considerably.

We concluded from our assumptions, and the median of the simulations, that the disease would be detected in a herd around days 13-19 after introduction. From the percentiles displayed in the results, it is obvious that there are some uncertainties in these values (Fig. 4). Another uncertainty is the value of $\rho$ (Fig. 2).

The probability of infection (1) is chosen such that it accounts for the distances in the housing structure. As the parameter estimates are based on data, which in this case originate from an experimental setup with at most one adjacent pen, this does not provide any information about the transmission patterns of ASFV in a realistic housing structure as simulated. Therefore, experiments providing data for larger housing structures would be relevant in order to estimate the parameters in a more realistic model.

Some experimental infection studies focus on using only one strain of the virus, which can be a limited factor as other isolates may have different clinical manifestation and spread behaviour. De Carvalho Ferreira 
et al. [24] estimated the transmission rate for the Malta '78 and the Netherlands '86 ASFV isolates and showed that the transmission rates were different for the two strain using the same criteria to define infectiousness and moment of infection.

It is important to mention that the results of our study represent a housing system of an industrialized swine production system. Thus, our findings might not be a good representation of within herd spread of ASF in herds in the Eastern European countries. This is because the housing structure of many herds in Eastern European countries might differ substantially from the housing structure of industrialized swine production systems in Western Europe.

\section{ACKNOWLEDGEMENTS}

The authors acknowledge Claire Guinat for a constructive dialog regarding how her animal experiments were performed.

\section{DECLARATION OF INTEREST}

None.

\section{REFERENCES}

1. Halasa T, et al. Control of an African swine fever epidemics in industrialized swine populations. Veterinary Microbiology 2016a; 197: 142-150.

2. Gallardo C, et al. Recombinant antigen targets for serodiagnosis of African swine fever. Clinical and Vaccine Immunology 2009; 16: 1012-1020.

3. Gallardo MC, et al. African swine fever: a global view of the current challenge. Porcine Health Management 2015; 1: 21. doi: 10.1186/s40813-015-0013-y.

4. Guinat $\mathbf{C}$, et al. Transmission routes of African swine fever virus to domestic pigs: current knowledge and future research directions. Veterinary Record 2016; 178: 262-267.

5. EFSA Panel. Scientific opinion on African swine fever. EFSA Journal 2014; 12: 3628.

6. Halasa T, et al. Simulating the epidemiological and economic effects of an African swine fever epidemic in industrialized swine populations. Veterinary Microbiology 2016b; 193: 7-16.

7. Halasa T, Toft N, Boklund A. Improving the effect and efficiency of FMD control by enlarging protection or surveillance zones. Frontiers in Veterinary Science 2015; 2: 70. doi: 10.3389/fvets.2015.00070.

8. Boklund A, et al. Comparing control strategies against foot-and-mouth disease: will vaccination be costeffective in Denmark? Preventive Veterinary Medicine 2013; 111: 206-219.

9. Roche SE, et al. How do resources influence control measures during a simulated outbreak of foot and mouth disease in Australia? Preventive Veterinary Medicine 2014; 113: 436-446.

10. Nigsch A, et al. Stochastic spatio-temporal modeling of African swine fever spread in the European Union during the high risk period. Preventive Veterinary Medicine 2013; 108: 262-275.

11. Backer JA, Hagenaars TJ, Van Roermund HJW. Modelling the effectiveness and risk of vaccination strategies to control classical swine fever epidemics. Journal of the Royal Society Interface 2009; 6: 849-861.

12. Martínez-López B, et al. A novel spatial and stochastic model to evaluate the within- and between-farm transmission of classical swine fever virus. I. General concepts and description of the model. Veterinary Microbiology 2011; 147: 300-309.

13. Thulke HH, Eisinger D, Beer M. The role of movement restrictions and pre-emptive destruction in the emergency control strategy against CSF outbreaks in domestic pigs. Preventive Veterinary Medicine 2011; 99: 28-37.

14. Guinat $\mathbf{C}$, et al. Dynamics of African swine fever virus shedding and excretion in domestic pigs infected by intramuscular inoculation and contact transmission. Veterinary Research 2014a; 45: 93-101.

15. Guinat $\mathbf{C}$, et al. Experimental pig-to-pig transmission dynamics for African swine fewer virus, Georgia 2007/ 1 strain. Epidemiology and Infection 2015; 144: 25-34. doi: $10.1017 / \mathrm{S} 0950268815000862$.

16. Pietschmann J, et al. Course and transmission characteristics of oral low-dose infection of domestic pig and European wild boar with a Caucasian African swine fever virus isolate. Archives of Virology 2015; 160: 1657-1667.

17. de Carvalho Ferreira HC, et al. Quantification of airborne African swine fever virus after experimental infection. Veterinary Microbiology 2013; 165: 243-251.

18. Anderson RM, May RM. Infectious Diseases of Human Dynamics and Control. Oxford: Oxford University Press, 1991.

19. Eblé $\mathbf{P}$, et al. Quantification of within- and between-pen transmission of foot-and-mouth disease virus in pigs. Veterinary Research 2006; 37: 647-654.

20. Velthuis AGJ, et al. Design and analysis of an Actinobacillus pleuropneumoniae transmission experiment. Preventive Veterinary Medicine 2003; 60: 53-68.

21. Pawitan Y. In All Likelihood: Statistical Modelling and Inference using Likelihood. Oxford: Oxford University Press, 2001.

22. Pitman J. Probability. New York: Springer Verlag, 1993.

23. Guinat $\mathbf{C}$, et al. Experimental pig-to-pig transmission dynamics for African swine fewer virus, Georgia 2007/1 strain-CORRIGENDUM. Epidemiology and Infection. 2016; 144: 2564-3566.

24. de Carvalho Ferreira HC, et al. Transmission rate of African swine fever virus under experimental conditions. Veterinary Microbiology 2013; 165: 296-304. doi: $10.1017 / \mathrm{S} 0950268816001667$.

25. Olsevskis E, et al. African swine fever virus introduction into the EU in 2014: experience of Latvia. Research in Veterinary Science 2016; 105: 28-30. 